\title{
Background and Bleaching Equivalence in Steady-State Adaptation of Vertebrate Rods
}

\author{
K. N. Leibovic, ${ }^{1}$ J. E. Dowling, ${ }^{2}$ and Y. Y. Kim ${ }^{1}$ \\ ${ }^{1}$ Department of Biophysics, State University of New York/Buffalo, Buffalo, New York 14222, and ²Biological Laboratories, \\ Harvard University, Boston, Massachusetts 02138
}

\begin{abstract}
We have investigated background and bleaching adaptation in vertebrate rods by intracellular recording in the retina of Bufo marinus. Backgrounds and bleaching produce adaptation in photoreceptors and lead to a shift and a compression of the response operating range. Threshold elevation due to backgrounds follows the Rose-DeVries rule at low intensities and the Weber-Fechner rule at high intensities. Threshold elevation due to bleaching is linear almost up to $17 \%$ bleached pigment and exponential thereafter.

An equivalence can be established between bleaching and backgrounds with respect to threshold elevation, on the one hand, and with respect to response compression, on the other. These equivalences are the same within experimental error. The equivalence, moreover, appears to extend to the complete response curve. These results have implications for psychophysics as well as for photoreceptor transduction.
\end{abstract}

At any given level of adaptation, vertebrate rods operate over a range of light intensities covering some 2-3 orders of magnitude. As the prevailing level of illumination changes, rods can shift this operating range over 7 or more orders of magnitude of light intensity. In the process, the stimulus threshold rises and the response amplitude is decreased. The state of adaptation of a rod is determined not only by the background light, but also by the state of photopigment bleaching.

Psychophysical experiments have established an interrelationship between bleaching and backgrounds in visual adaptation (Crawford, 1947). For each level of bleaching there is an equivalent background that raises the threshold by the same amount under a variety of different stimulus conditions (Blakemore and Rushton, 1965).

Although adaptive control occurs at various levels of the visual pathway, most of it depends on events at the initial stages of the sensory process (Boynton and Whitten, 1970; Dowling and Ripps, 1972) and, in fact, must be assigned to the photoreceptors. For, second-order neurons can operate only over some 3 orders of magnitude of stimulus intensity and could not accommodate the enormous range of visual adaptation even if one were to take account of the possibilities of neuronal mod-

Received June 16, 1986; revised Oct. 27, 1986; accepted Oct. 31, 1986

This work was supported by Grants 5R01EY03672 from the National Eye Institute and C6602007 from NIH-PHS Biomedical Research Support of SUNY/ Buffalo. We received the generous help of our colleagues Paul Brown, Mike Zeldin, and Malcolm Slaughter. Z. H. Pan also assisted with some of our data analyses.

Correspondence should be addressed to K. N. Leibovic at the above address.

Copyright (C) 1987 Society for Neuroscience $0270-6474 / 87 / 041056-08 \$ 02.00 / 0$ ulation and accommodation. The capabilities for adaptation must exist at the input if they are to be transmitted to subsequent stages of the system (Leibovic, 1971). Extracellular and intracellular recordings from photoreceptors are consistent with this view (Normann and Werblin, 1974; Grabowski and Pak, 1975, Kleinschmidt and Dowling, 1975; Fain, 1976; Pepperberg et al., 1978).

In this paper we present a comparison of the effects of bleaching and backgrounds on the shift of the operating range and on response compression, and we consider the possible equivalences between backgrounds and bleaching in this context. We have recorded the intracellular responses in the isolated retina, which is particularly suited to bleaching studies. In this preparation the pigment does not regenerate after bleaching. The threshold is permanently elevated, and one can study light and dark adaptation in the steady state (Weinstein et al., 1967). Our results lead us to conclude that there is indeed an equivalence between bleaching and backgrounds with respect to the parameters studied in this paper. This does not mean that the equivalence is universal: It clearly does not hold with regard to the response waveforms whose kinetics in the presence of backgrounds are distinctly accelerated by comparison with bleaching. Nor does the equivalence hold with regard to temporal summation and other kinetic parameters we are studying at present.

\section{Materials and Methods}

The experiments were performed at Harvard University and at the State University of New York at Buffalo.

Dissection. Bufo marinus was dark-adapted for at least $3 \mathrm{hr}$ before decapitation. Dissection was performed in dim red light. The eyes were enucleated, the anterior portion cut away, the posterior portion sectioned, and the retina peeled off, free of pigment epithelium, in toad Ringer: $108 \mathrm{mM} \mathrm{NaCl}, 2.5 \mathrm{~mm} \mathrm{KCl}, 1.6 \mathrm{mM} \mathrm{CaCl}_{2}, 0.6 \mathrm{mM} \mathrm{Na}_{2} \mathrm{SO}_{4}$, $1.2 \mathrm{mM} \mathrm{MgSO}_{4}, 0.13 \mathrm{~mm} \mathrm{NaHCO}, 5.6 \mathrm{~mm}$ glucose, $3 \mathrm{~mm}$ HEPES buffered to $\mathrm{pH}$ 7.4. The isolated retina was placed on a piece of filter paper, receptor side up, and transferred to a preparation holder containing Ringer's solution.

Instrumentation. The electrophysiological instrumentation and optical stimulus system were similar to one previously described (Kleinschmidt and Dowling, 1975). Cell penetrations were achieved with fine glass pipettes filled with $3 \mathrm{M} \mathrm{K}$-acetate $+1 \mathrm{M} \mathrm{KCl}$ and having a resistance of 200-250 M . Simultaneously with the intracellular recordings, the ERG was monitored with an $\mathrm{Ag}-\mathrm{AgCl}$ electrode. The responses were displayed on an oscilloscope and recorded on a strip-chart recorder. The optical system contained 2 light paths with interposed $505 \mathrm{~nm}$ interference filters, KG3 heat filters, and neutral density (ND) filters or wedge by means of which the light intensity could be varied. One path was used either for bleaching the retina or to project a steady background light onto it, while the other was used to deliver $100 \mathrm{msec}$ flashes, which served as the stimulus. In all cases the whole preparation was evenly illuminated by light. When not attenuated by ND filters, the background beam at SUNY/Buffalo delivered $1.95 \times 10^{14} \mathrm{photons} / \mathrm{cm}^{2} / \mathrm{sec}$, while 
the stimulus beam delivered $8 \times 10^{12}$ photons $/ \mathrm{cm}^{2} / \mathrm{sec}$ onto the preparation. The light fluxes at Harvard were comparable. At both Harvard and SUNY/Buffalo the photon fluxes were measured with a calibrated photocell at the position of the preparation. In this paper all values are standardized with respect to the calibrations at SUNY/Buffalo. Thus, a $100 \mathrm{msec}$ flash in the stimulus path attenuated by $5 \mathrm{ND}$ units contains $8 \times 10^{6}$ photons $/ \mathrm{cm}^{2}$.

Bleaching. The flash stimuli and the backgrounds we used during the course of an experiment produced negligible bleaching. In those experiments in which the retina was to be bleached to a desired level, we turned on the unattenuated background light for a predetermined time. The amount of pigment bleached by this procedure was determined in a separate series of experiments as follows: The tissue was placed in a special holder between 2 coverslips and positioned exactly as in the electrophysiological experiments. The background light was then turned on at a chosen intensity and for varying durations. A Cary or Beckman spectrophotometer was used to obtain the absorption spectra of the freshly isolated, dark-adapted retina and of the same retina after each bleaching exposure. Finally, the retina was bleached to completion and its absorption spectrum was obtained once more. The fraction of pigment bleached on each exposure could then be calculated from the difference spectra.

We found that the data agreed closely with the theory developed earlier for the isolated retina (Leibovic, 1975, 1983). For the unattenuated background path of $1.95 \times 10^{14}$ photons $/ \mathrm{cm}^{2} / \mathrm{sec}$ at SUNY/Buffalo, the bleached fraction, $B(t)$, after time $t$ (sec) of light exposure is given accurately by the equation

$$
\begin{aligned}
B(t)= & 1-0.9971 \exp (-0.00513 t) \\
& -0.0029 \exp (-0.06193 t) .
\end{aligned}
$$

Equation (1) was therefore used to determine the exposure time for any desired amount of bleaching in our experiments.

Leakage during intracellular recording. During intracellular recording it is difficult, if not impossible, to prevent some leakage between the electrode and the cell membrane. This problem is aggravated in the presence of a bright background and during bleaching with high-intensity lights. For one, the cell is not a static structure: Even as the clectrode is advanced in contact with the membrane, a flash of light often leads to penetration, presumably because the membrane moves concomitantly with the light response. Leakage can be a serious problem in longterm recordings, especially those involving bright backgrounds and bleaching. As shown below, a consistent reduction of response amplitude was observed in the presence of a background and after bleaching. But this is not simply due to leakage, as is clear from the following finding: Several cells were usually penetrated both before and after bleaching or in the presence of a background. The response amplitudes were always larger before than after bleaching or while the background light was on, and the greater the extent of bleaching or the more intense the background, the smaller the responses. On the other hand, when reduced responses were recorded from a cell after bleaching or in the presence of a background, it was sometimes found, on penetrating a neighboring cell, that the responses were significantly larger than in the first cell. In such cases it was assumed that excessive leakage had occurred in the first cell and its responses were discarded.

The basic experiment. After penetrating a cell, its dark-adapted responses from threshold to saturation were determined with a series of flashes of increasing intensity. In the experiments involving backgrounds, the background light was turned on and after the cell had reached a steady state, a new response series was similarly determined. In the experiments involving bleaching, the bleaching light was turned on for a precisely preset time. After the light was turned off and the cell had recovered and reached a steady state, a response series was again determined. In all cases we then plotted maximum response amplitude versus flash intensity, i.e., the "operating curve." From these figures it was possible to obtain rise of threshold and saturated response amplitude as functions of background and bleaching. At high flash intensities, at which the response exhibits a "spike" followed by a "plateau," we plotted both spike and plateau amplitudes. For the saturated responses we have used the average of spike and plateau as a measure of the saturated amplitude.

Statistical treatment of the data. There are several sources of variability in the data. (1) There is the usual "noise" in the recordings, which is relatively small. (2) During the course of an experiment the responsiveness of a cell can change: It may increase, perhaps due to recovery from injury or trauma resulting from the dissection or cell penetration,

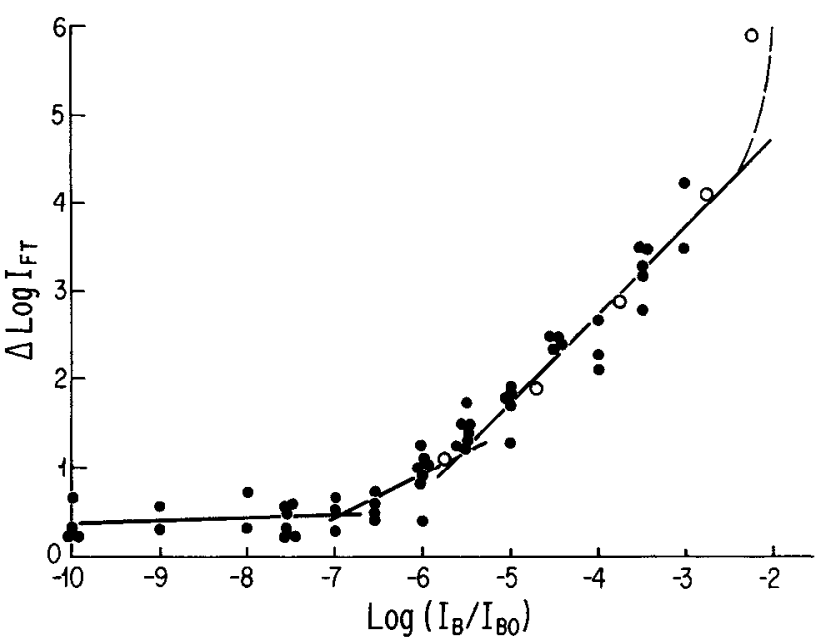

Figure 1. Threshold elevation versus background. The ordinate is the $\log$ of threshold elevation, $I_{\mathrm{FT}}$ being the threshold flash intensity. The abscissa is the neutral density attenuation, ND, of the background beam, with $I_{B 0}$ the unattenuated and $I_{B}$ the attenuated intensity. Least-squares regression lines have been fitted as follows: ND -10 to $-7: y=0.69+$ $0.3 x ; \mathrm{ND}-7$ to $-6: y=3.93+0.50 x$; and ND -5 to $-3: y=6.77+$ $1.00 x$. Filled circles, present study; open circles, data from Kleinschmidt and Dowling (1975).

or it may decrease due to deterioration of the cell; in some cases, responsiveness can also improve during long-term recording after a temporary deterioration. (3) In experiments involving backgrounds and bleaching, possible cell movement and leakage can be additional sources of variability. (4) The effective photon catch with a given background or bleaching exposure will depend on the orientation of the photoreccptors in rclation to the incident light beam. (5) With bright backgrounds and prolonged bleaching, the cells are tested to their limits. This is even more the case when an impaled cell is stimulated with saturating flashes after such exposures. As a result, cells are often lost after such intense illumination. In some cases we then penetrated other cells to determine thresholds and response amplitudes after bleaching. Differences in cell responsiveness represent an additional source of variability. Nevertheless, we believe that it is appropriate to include such data for 2 reasons: First, the responsiveness of successive cells may fluctuate up or down, so that no systematic bias should arise; second, the possibly better responsiveness of new cells compensates for the likelihood of the poorer responsiveness of the cell that was impaled during the bleaching or background exposures.

After examining the data points we used least-squares regression where a linear fit to the data secmed appropriate. This was done for the different ranges of threshold elevation as a function of bleaching and backgrounds in Figures 1 and 2. In cases of response compression there is more scatter in the data for the reasons mentioned. In addition, linear relationships provide only poor approximations. But we could obtain improved statistics by the following procedure: The relative response amplitude $A$ is defined as the ratio of the saturated response amplitude after bleaching, or in the presence of a background, to the original darkadapted response. We divided the range of $A$ into sets of overlapping intervals and for each interval calculated the mean and SD of the percentage bleached, $B$, or of the background ND (neutral density $\log \left[I_{B} /\right.$ $I_{B 0}$ ], where $I_{B}$ and $I_{B 0}$ are the intensities of the attenuated and unattenuated background, respectively). We then repeated the procedure by dividing the ranges of $B$ and of the background ND into overlapping intervals and calculating the means and SDs for $A$. The resulting values provide better estimates of $A$ as a function of $B$ and $\left(I_{B} / I_{B 0}\right)$. The points of Figures 3 and 4 were obtained by this procedure.

\section{Results}

\section{Dependence of threshold on backgrounds}

When a background light is projected onto the retina, the steadystate threshold is elevated in the manner shown in Figure 1, taking a $0.5 \mathrm{mV}$ response of the recorded membrane potential 


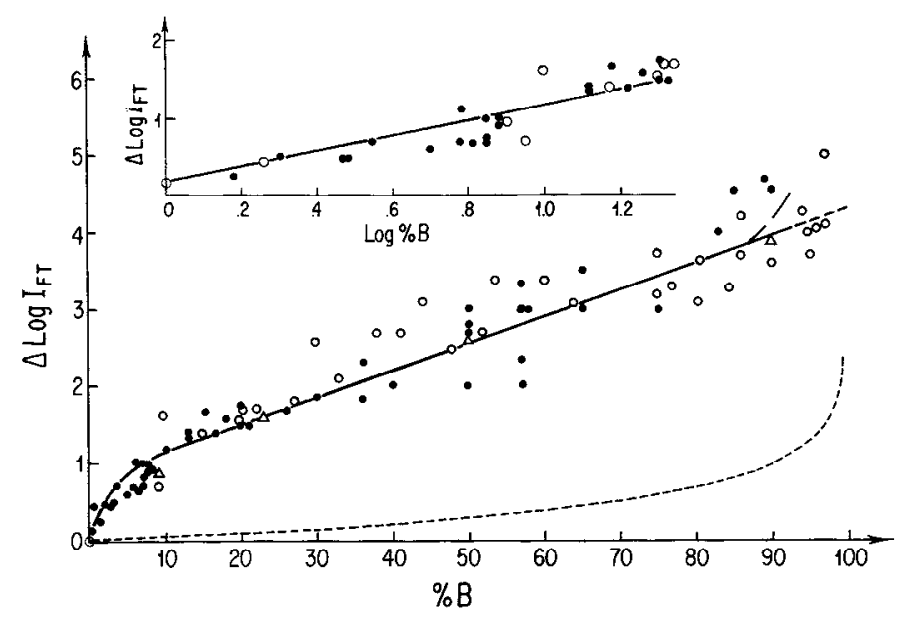

Figure 2. Threshold elevation versus bleaching. The ordinate is as in Figure 1; the abscissa plots the percentage of bleached pigment. Filled circles, present study; open circles, data from Pepperberg et al. (1978); and open triangles, data from Cornwall et al. (1983). The inset replots the data points up to $20 \%$ bleached pigment on double-logarithmic coordinates. The least-squares regression lines on the inset and the main plot are, respectively: $0 \% \leq B \leq 20 \%: y=0.2+x$; and $18 \% \leq B \leq$ $85 \%: y=0.8+0.035 x$. The first translates into $I_{\mathrm{FT}} \propto B$ and the second into $I_{\mathrm{FT}} \propto \exp (0.08 B)$. The dotted curve plots the threshold elevation if it were due solely to the photon absorption probability of the unbleached fraction of rhodopsin.

as the threshold criterion. The abscissa is the neutral density attenuation (ND) of the background light (unattenuated flux, $1.95 \times 10^{14}$ photons $/ \mathrm{cm}^{2} / \mathrm{sec}$ at $505 \mathrm{~nm}$ ). The ordinate is the elevation of threshold flash intensity (in ND units) above the dark-adapted threshold intensity. The latter was generally between 5.3 and 6.6 ND units below the unattenuated, $100 \mathrm{msec}$ stimulus flash of $8 \times 10^{11}$ photons $/ \mathrm{cm}^{2}$ at $505 \mathrm{~nm}$. Thus, the dark-adapted threshold response required between 0.06 to 1.1 photons per rod (for a rod diameter of $6 \mu \mathrm{m}$ ). This should be understood as a mean value in the presence of rod-to-rod coupling (Gold, 1979), which increases the effective area for photon capture, and photon (Hecht et al., 1942) and intrinsic photoreceptor fluctuations (Baylor et al., 1979), which produce response variability.

Figure 1 shows our data points, as well as those of Kleinschmidt and Dowling (1975, Fig. 10) from Gecko rods. It is cvident that these 2 sets of data recorded intracellularly in 2 different species are in good agreement with each other. Similar results have been obtained by others (e.g., Grabowski and Pak, 1975; Fain, 1976, Fig. 9; Baylor et al., 1980, Fig. 15, using suction electrode current recording). The lines drawn through the points are least-squares regression lines. They have slopes of close to zero at low background intensities, 0.5 at intermediate, and 1 at high background intensities. Thus, the threshold increases little at low background intensities. At higher intensities, the threshold can be approximated by the Weber-Fechner rule, according to which threshold increases linearly with background. In the transition between these 2 regions, the threshold can be approximated by the Rose-DeVries rule, which requires the threshold to vary as the square root of background intensity. At the highest intensities, the rods saturate and the threshold rises without limit. Thus, in Figure 1,

$$
I_{\mathrm{FT}} \approx \text { constant, } \log \left(I_{B} / I_{B 0}\right) \leq-7
$$

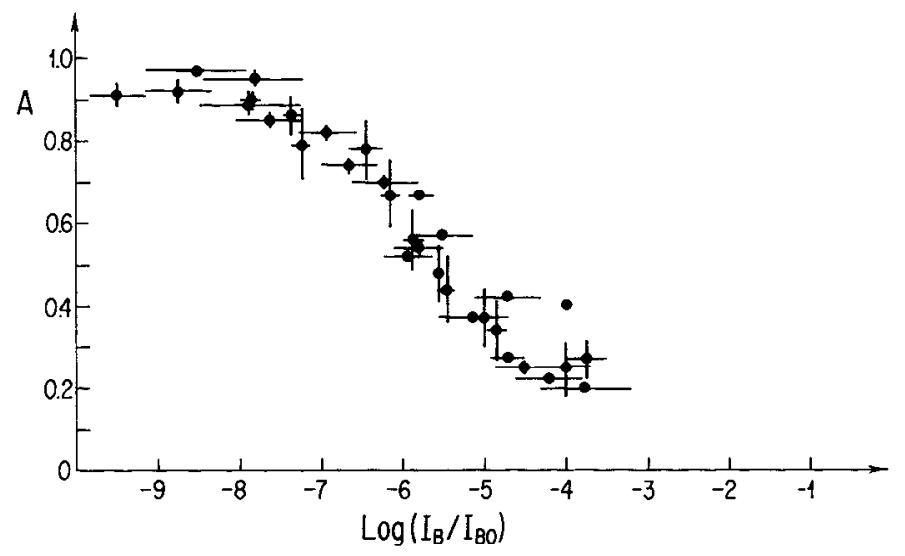

Figure 3. Response compression versus background. $A$ is the ratio of the saturated response amplitude in the presence of a background to the dark-adapted amplitude. The data points are mean values, and the lines through the points are one-half SD on each side of the points. The $A$-axis was divided into overlapping strips as follows: $0 . k \leq \Delta A \leq$ $0 .(k+1)$ and $0 . k 5 \leq \Delta A \leq 0 .(k+1) 5$. Within each strip we calculated means and SDs of the background intensities from the raw data; the background NDs were then grouped into overlapping subsets and the means and SDs of $A$ calculated similarly. The data for both the horizontal and vertical strips (or subsets) are plotted.

$$
\begin{aligned}
I_{\mathrm{FT}} \propto & \sqrt{I_{B}},-7 \leq \log \left(I_{B} / I_{B 0}\right) \\
& \leq-5.75(\text { Rose-DeVries }) \\
I_{\mathrm{FT}} \propto & I_{B},-5.75 \leq \log \left(I_{B} / I_{B 0}\right) \\
& <-2.5 \text { (Weber-Fechner) }
\end{aligned}
$$

where $I_{\mathrm{FT}}$ is the threshold flash intensity, $I_{B}$ the attenuated background intensity, and $I_{B 0}$ the unattenuated background intensity.

It is noteworthy that both the range of background intensities and the rise of threshold over that range are comparable in the intracellular recordings from $B u f o$ rods and the psychophysical data from human subjects (cf. Blakemore and Rushton, 1965, Fig. 2). By contrast, rods of rats (Penn and Hagins, 1972), mudpuppy (Normann and Werblin, 1974), and Macaca fascicularis (Baylor et al., 1984) show saturation at much lower background intensities. In these species - unlike Bufo, frog, and Gecko-the rod response due to background does not recover appreciably after the initial transient.

\section{Dependence of threshold on bleaching}

Figure 2 shows how the threshold varies with bleaching. The rise in the logarithm of flash intensity is plotted against the percentage bleached. On the same graph we have plotted the data points of Pepperberg et al. (1978), which were obtained from extracellular recordings in the skate retina; we have also included the data of Cornwall et al. (1983) obtained with a suction electrode from tiger salamander. Again, there is good agreement of these data from different species acquired by different techniques. Figure 2 shows that the threshold varies exponentially from $20 \% B$ to about $85 \% B$ and thereafter rises to saturation at $100 \% B$. In the inset to Figure 2 we have replotted our data on double-logarithmic scales. This shows that the threshold varies approximately linearly with bleaching up to some $17 \% B$. Accordingly, in Figure 2,

$$
\begin{aligned}
& I_{\mathrm{FT}} \propto B, 0 \% \leq B<17 \% \\
& I_{\mathrm{FT}} \propto \exp (0.08 B), 20 \% \leq B \leq 85 \% .
\end{aligned}
$$




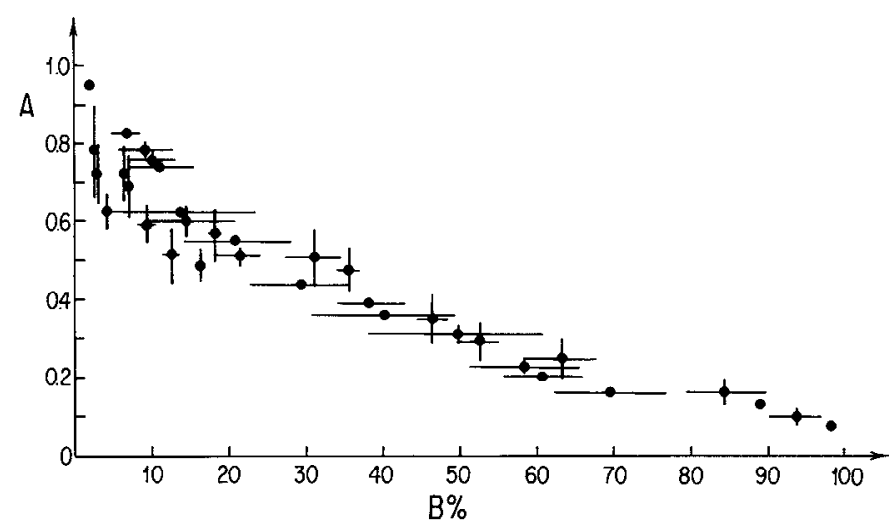

Figure 4. Response compression versus bleaching. Data analyzed by the same method as in Figure 3.

A linear variation of threshold with bleaching up to $17 \% \mathrm{~B}$ has not been reported previously (but see Backstrom and Hemila, 1979), but our data, as well as a reexamination of the data of Pepperberg et al., are in agreement on this point.

\section{Compression of response amplitude with background and bleaching}

As the flash intensity is increased the response amplitude, for any state of adaptation, finally saturates at its maximum value. If the flash intensity is increased beyond that, the response decreases somewhat. The saturated response displays a characteristic spike and plateau. We have taken the average of maximal spike and plateau as a measure of the saturated response amplitude. When this amplitude, either in the presence of a background or after bleaching, is divided by the corresponding response amplitude of the dark-adapted cell, we obtain the relative reduction of response amplitude $A$.

As explained in Materials and Methods, these experiments have inevitable scatter of the data associated with them. An inspection of the data does not suggest any obvious linear relationships. Accordingly, we performed the analysis as described in Statistical Treatment of the Data. The results are shown in Figure 3 for $A$ as a function of background and in Figure 4 for $A$ as a function of bleaching.

\section{Equivalences between backgrounds and bleaching}

There is, ideally, 1 background $I_{B}$ and 1 bleached fraction $B$ that produce a given threshold elevation, $\Delta I$. In that sense, $I_{B}$, $B$ are equivalent with respect to $\Delta I$. Similarly, an equivalence between $I_{B}$ and $B$ can be established with respect to amplitude reduction $A$. In practice, scatter of the experimental points will result in a mean value and SD of $I_{B}$ and $B$ for any given value of $\Delta I$ or $A$. We calculated these mean values using the same procedure as in Figures 3 and 4: We divided the threshold range into overlapping intervals and found the mean and SD of $I_{B}$ and of $B$ in cach interval. In each interval the equivalent $I_{B}, B$ pair is given by their mean values. Similarly, we found the equivalent $I_{B}, B$ pairs for amplitude reduction $A$.

The results are plotted on Figure 5. There is no apparent difference between the 2 families of points, one representing the equivalence with respect to thresholds and the other with respect to amplitudes. Each point of one family is within at most a half SD of a point from the other family.

It should be noted that the above method does not depend

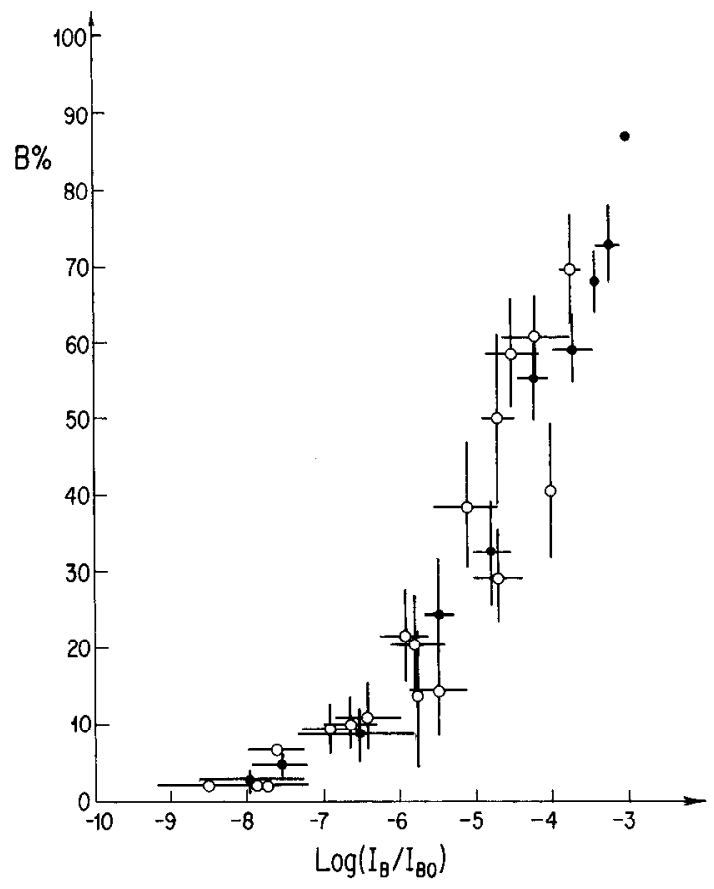

Figure 5. Bleaching and background equivalences. The procedure used in Figures 3 and 4 leads to an equivalent $I_{B}, B$ pair for each strip $\Delta A$. A similar procedure can be applied to the threshold data of Figures 1 and 2 . Here, the threshold axis was divided into the overlapping strips $k \leq \log I_{\mathrm{FT}} \leq k+1$ and $k .5 \leq \log I_{\mathrm{FT}} \leq(k+1) .5$. Then, for each such strip we get another equivalent $I_{B}, B$ pair. The means together with the SDs obtained in this way are plotted. The filled circles are threshold equivalent pairs; open circles, amplitude equivalent pairs.

on any curves drawn by eye. The points on Figure 5 are derived from the raw data and represent objective statistical estimates. The procedure used here is, however, different from the linear regression used in Figures 1 and 2. Therefore, there need not be exact agreement between Figure 5, on the one hand, and Figures 1 and 2 or Figures 3 and 4, on the other hand.

Based on our analysis we can accept the proposition that the equivalences of $I_{B}$ and $B$ with respect to $\Delta I$ and to $A$ are the same, at least to a first approximation.

\section{Possible extension of the $\left(\mathrm{I}_{\mathrm{B}}, \mathrm{B}\right)$ equivalence}

Having found that threshold elevation and amplitude reduction generate the same equivalence between $I_{B}$ and $B$, at least within the statistical variability of the experiments, we can ask whether this equivalence extends to the complete response curve. For, threshold and amplitude reduction refer to the beginning and end of the response curve, respectively. If there is a relationship including these end points, that relationship may also apply to intermediate points. To explore this question we have, however, to keep in mind the variability of the data. Thus, an equivalence, say, between a ND 6 background and 10\% bleaching applies to mean values with SD that may range from 7 to almost 5 ND in $I_{B}$ and from 5 to $20 \%$ in $B$. Therefore, in any single experiment we may not find good agreement in the response curves for any specific mean values of $I_{B}$ and $B$. Fortunately, there is a way out of this difficulty. For, we are looking at the agreement of the intermediate points on the response curves, when the end points coincide. Any deviations of the end points from their mean values should also apply to intermediate points. We have therefore chosen to look for the overall shapes of the response 

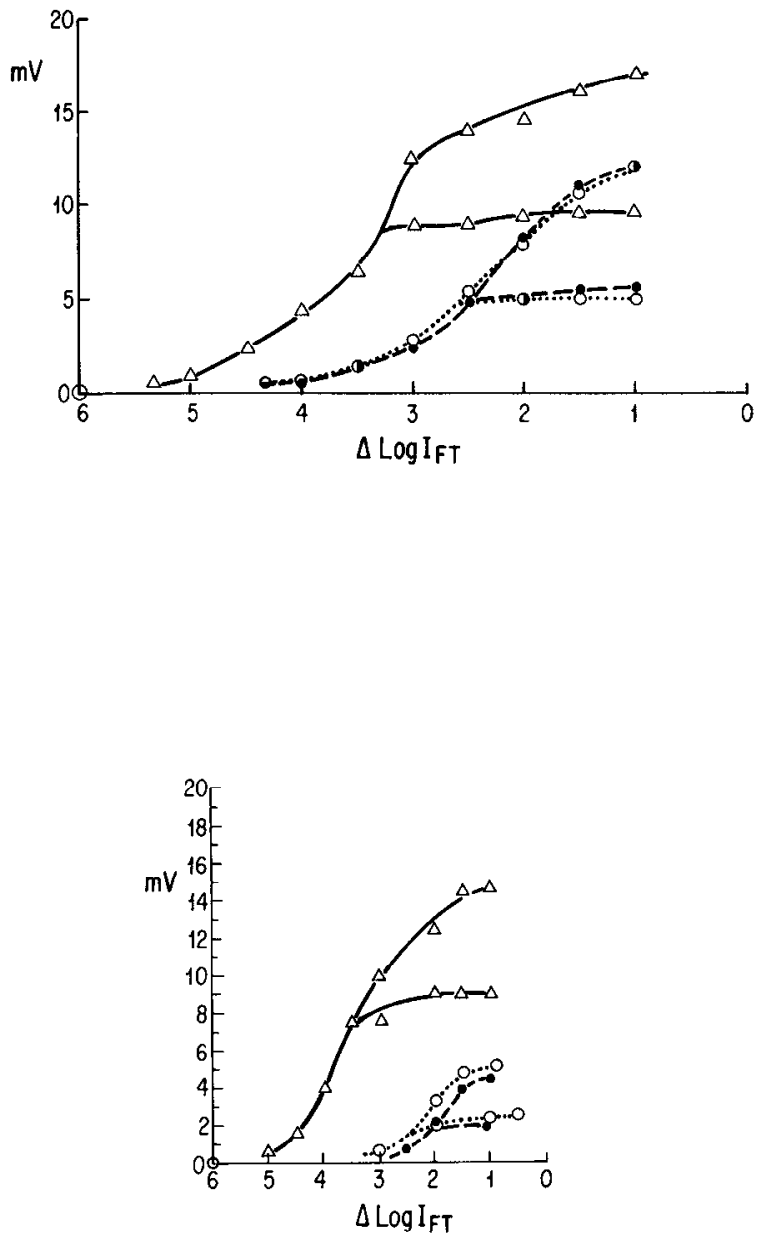
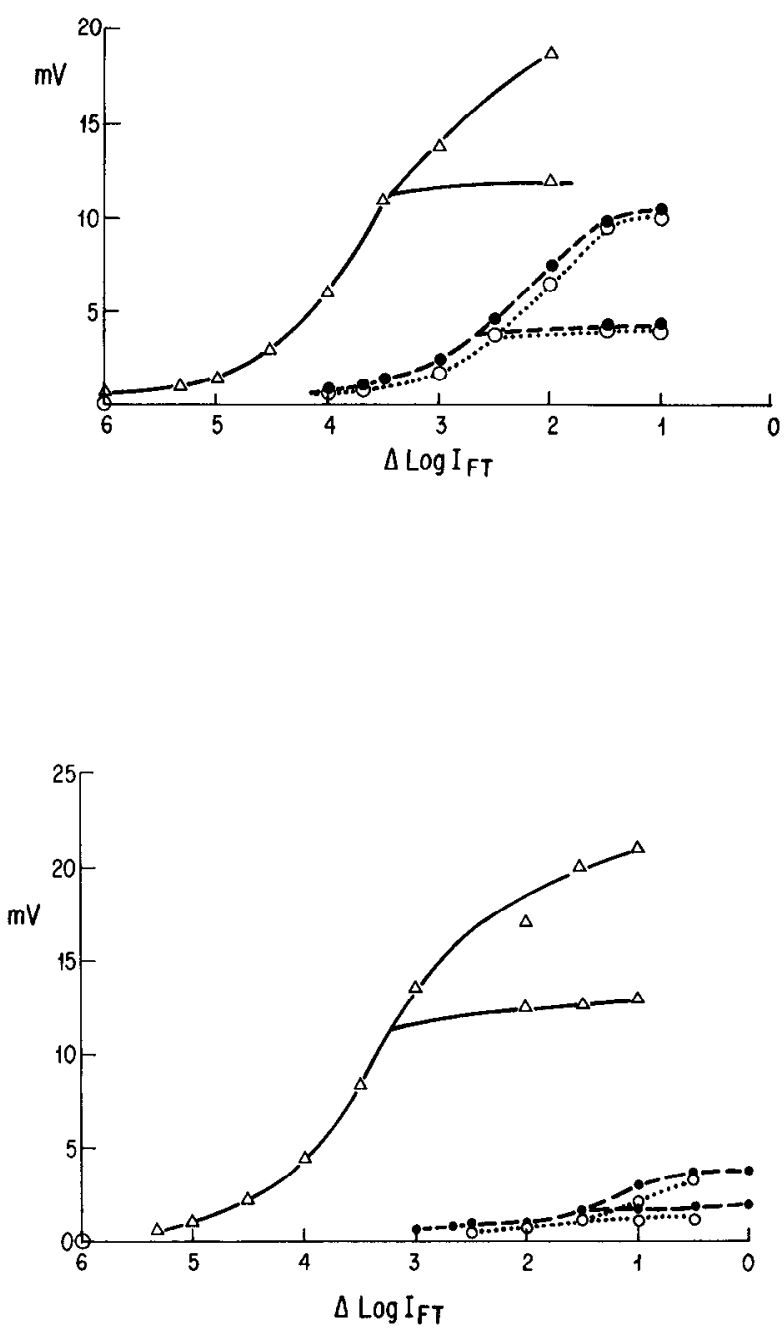

Figure 6. Bleaching and background equivalent response curves. Four examples are shown for near coincidence of the response curves for different background and bleaching pairs. The highest curve plots peak responses (in $\mathrm{mV}$ ) versus flash intensity (in relative ND units) for the dark-adapted cell. The open circles show the response curve in the presence of a background; closed circles, responsc curve after blcaching. Each curve splits into two branches at high intensities, one for the spike and the other for the plateau. Upper left, Comparison of a ND $=6$ background with a $10 \%$ bleached retina; upper right, $\mathrm{ND}=5$ and $21 \%$ bleached retina; lower left, ND $=4$ and $48 \%$ bleached retina; lower right, ND $=3.5$ and $70 \%$ bleached retina. The close agreement at threshold and at saturation is reflected in the near coincidence of the curves.

curves when they coincide or are close to each other at threshold and at maximum amplitude.

Figure 6 shows the results of 4 experiments in which backgrounds and bleaching differ widely. In each of the 4 cases the $I_{B}, B$ pairs fall within the range to qualify as equivalent with respect to $\Delta I$ and $A$ in Figure 5. The threshold elevations and amplitude reductions are close to the expected values. As these figures indicate, the closeness at the end points carries over to the complete response curve.

Figure 6, upper left, shows a response curve for the darkadapted state, another for the dark-adapted retina with a background of ND 6, and a third for the retina after being bleached by $10 \%$. The last 2 response curves are virtually identical. In Figure 6, upper right, we have a similar comparison of a background ND 5 and 21\% bleaching. In Figure 6, lower left, a background of ND 4 is compared with $48 \%$ bleaching, and in Figure 6, lower right, a 3.5 ND background with $70 \%$ bleaching. The closeness of the end points of the curves in Figure 6, upper left and right, are within the experimental scatter of the equivalences of $I_{B}$ and $B$ from Figure 5, and they result in the close agreement of the complete response curves. In Figure 6, lower left, the separation between the response curves parallels the greater deviation of $I_{B}(\mathrm{ND}=4)$ and $B(48 \%)$ from the mean values of Figure 5. But, as expected, both threshold elevation and response compression are smaller for $I_{B}$ than for $B$, and the intermediate points on the response curves follow the same pattern. Similarly, Figure 6, lower right, displays response curves for the intense, 3.5 ND background and the high level of bleaching $(70 \%)$.

These illustrations are not from isolated cases, as we have observed this agreement repeatedly, though not invariably. The deviations from agreement may be due to variability among cells or to some of the other factors described in Materials and Methods.

\section{Discussion}

The idea of an equivalent background arose in psychophysics, first in the context of threshold elevation. Our results support the proposition that the equivalence with respect to threshold elevation is the same as that with respect to response compres- 

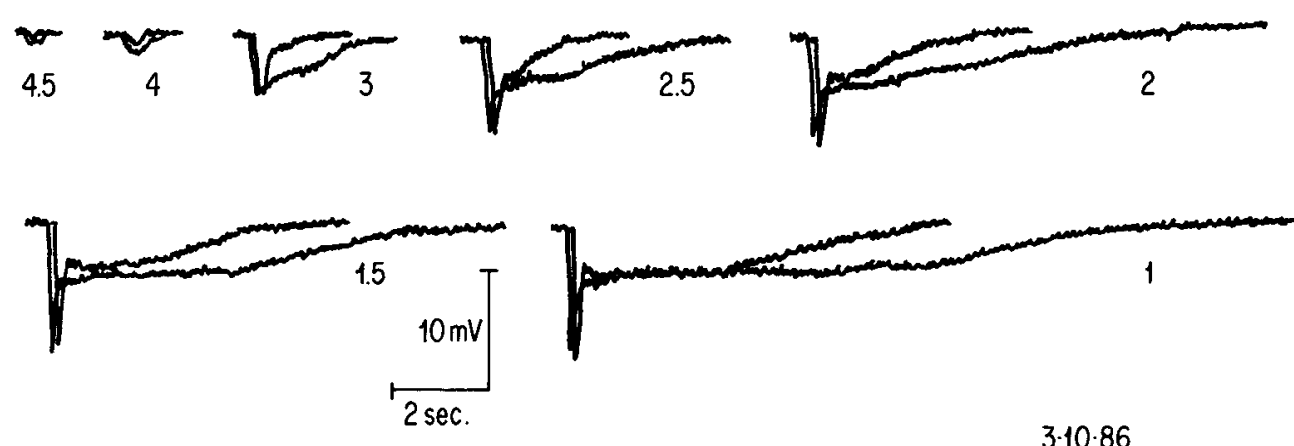

Fig. 7
Figure 7. Backgrounds and bleaching yield different response waveforms. Superimposed response waveforms to 100 msec flashes in the presence of a background $\log \left(I_{B} / I_{B 0}\right)=-5$ and after the equivalent $30 \%$ bleaching. The background responses are from 1 cell, the bleaching responses are averaged from 3 response series of 2 cells in the same piece of retina. The background responses are slightly displaced to the right of the bleaching responses for the sake of clarity. The relative flash ND is shown below each response pair. The responses in the presence of a background are of shorter duration than the responses after bleaching. sion and, moreover, that the equivalence cxtends to the complete operating curve. This has interesting implications for psychophysics, as well as for the transduction of the absorbed light to a neural response. We will discuss these matters after some comments on specific results.

\section{Thresholds}

Threshold elevation is synonymous with a shift of the operating curve to higher light intensities. This is one of the major attributes of adaptation. [It is graphically illustrated, for example, in Fig. 10 of Kleinschmidt and Dowling (1975) for background adaptation.] The similarities of our threshold data in Bufo with those of other investigators using different species shows that there are important functional parallels in vertebrate rods.

At low to intermediate background intensities there is appeal in the treatment of thresholds as a signal-and-noise problem (Leibovic, 1972, Chaps. 8, 19), and we have approximated thresholds in that range by the Rose-DeVries law (Rose, 1942; DeVries, 1943). At higher intensities the Weber-Fechner law operates.

Our threshold data on bleaching and comparison of these data with other results, especially those of Pepperberg et al. (1978), suggest that threshold elevation varies linearly with bleaching up to some $17 \%$ of bleached rhodopsin. Beyond that the rise is exponential with bleaching. The linear region is not immediately apparent due to experimental scatter, unless there are sufficient data points. This may be why it has not been reported previously, although there have been allusions to the possibility of linear portions of the curve (e.g., Pepperberg, 1984).

The threshold rises slowly with dim backgrounds and then more rapidly as background intensity increases. This is the reverse of the rise in threshold with bleaching, which is rapid up to some $15-20 \%$ and slows thereafter.

A test flash can be varied in duration and intensity. Therefore, a theshold flash could, in principle, provide 2 criteria for equivalence between background and bleaching. But, as we shall discuss elsewhere, the intensity and duration obey a form of Bloch's law, so that during a critical integration period the only significant variable is the number of photons in the flash. For this reason there can bc only 1 thrcshold equivalent relation for photoreceptors.

\section{Response compression}

Response compression is the second major attribute of rod adaptation. It is implicit in psychophysical data, since it is well known that rod responses are depressed and cone responses becomc dominant as vision changes from scotopic to photopic. Rod and cone response compression has been demonstrated on numerous occasions in both extra- and intracellular recordings (e.g., Boynton and Whitten, 1970; Dowling and Ripps, 1972; Normann and Werblin, 1974). Here we have addressed the separate relationships of response compression to backgrounds and bleaching, respectively (Figs. 3 and 4), and we have asked whether the equivalence that can be established with respect to saturated amplitudes is the same as that with respect to thresholds. We found close agreement of the 2 equivalence relations. We have reported this conclusion before (Leibovic, 1981, 1983b), and it is supported by the data of Clack and Pepperberg (1982, see their Fig. 7). They recorded the extracellular ERG of the aspartate-treated isolated retina of skate, which is rod dominated. Aspartate is known to suppress postreceptoral responses (e.g., Kleinschmidt and Dowling, 1975, Figs. 1 and 2) and to enhance and modify the receptor responses. They did not separate the effects of bleaching and backgrounds, as we have done in our Figures 3 and 4, but their Figure 7, despite the scatter of the data, shows no gross differences in the families of points derived from backgrounds and bleaching, respectively. Their Figure 6 is also in agreement with our proposition that the equivalence between bleaching and backgrounds at threshold and saturated amplitude extends to the complete response curve.

\section{Some implications for psychophysics}

We have already alluded to the similarities between the intracellular results and psychophysical data. The same Rose-DeVries and Weber-Fechner rules apply to similar threshold ranges of background adaptation, while in bleaching adaptation the exponential relationship (Dowling, 1960; Rushton, 1961) is obeyed over much of the range. In the latter case, the value of the exponent may not be the same. For example, Rushton (1961, 1962, Fig. 3) derived $\Delta I_{\mathrm{FT}} \propto \exp (46 B)$. He did this by extrapolating the log threshold data to the regeneration curve of a rod monochromat. Then, in order to fit an exponential from 100 to $0 \%$ bleaching, the log threshold must extend to about 20 (or ln threshold to about 46). The threshold actually traced by the rod monochromat extended only to about $7 \log$ units above the dark-adapted valuc. Similarly, in the normal cyc, the threshold elevation does not usually span $20 \log$ units. The precise values of the parameters thus depend in part on the treatment of the data. The intracellular data indicate that the rod threshold rises about $5 \log$ units for a background of $\mathrm{ND}=2$ or $95 \%$ bleaching approximately and that it rises to saturation thereafter. According to Rushton (1962, fig. 3), by comparison, the threshold 
would have risen almost $20 \log$ units. This is a large discrepancy. It might be worth exploring further the difficulties of measuring psychophysical thresholds at these high bleaching levels.

Barlow and Sparrock (1964) showed psychophysically that a background which produced the same threshold elevation as a given level of bleaching appeared equally as bright as the afterimage due to the bleaching. Thus, the psychophysical equivalence with respect to thresholds appears to be the same as that with respect to brightness perception. Our response curves plot the peak amplitudes of the flash responses while the Barlow and Sparrock data refer to quasi steady-state conditions. Our results showing the equivalence of the complete response curves, however, suggest that the perceived brightness of equal flashes on backgrounds and equivalent bleached areas may also be the same. But this would require that brightness perception be mediated by the peak photoreceptor response since only the peak amplitudes of the flash responses are the same. The remainder of the response waveforms in the presence of a background or the equivalent bleached fraction are quite different, as illustrated in Figure 7. It is interesting that the information on brightness may thus be conveyed in the complex waveform of photoreceptor responses by just one point that, theoretically, should also be the easiest to detect and transmit (Leibovic, 1986).

\section{Some implications for transduction}

An equivalence between backgrounds and blcaching over the complete response range raises the question of whether the same mechanisms may mediate the shift and compression of the operating curve in both conditions. To this end, let us consider a particular case-say $30 \%$ bleaching and the approximately equivalent background of ND 5, which contains some $1.95 \times$ $10^{9}$ photons $/ \mathrm{cm}^{2} / \mathrm{sec}$, or about 550 photons $/ \mathrm{rod} / \mathrm{sec}$. At this intensity it would take about $150 \mathrm{hr}$ to deliver a number of photons equal to $30 \%$ of the rhodopsin in a rod, and more than $600 \mathrm{hr}$ to actually bleach $30 \%$ of the rhodopsin in our experimental setup. This calculation assumes a rod diameter of $6 \mu \mathrm{m}$ and $10^{9}$ rhodopsin molecules per rod. Thus, as a rough estimate, some 170 rhodopsin molecules/sec will be photolyzed by this background, and the percentage bleached during an experiment will be quite negligible. Yet the photolysis of 170 molecules/sec has the same effect on threshold elevation and response compression as the presence of $3 \times 10^{8}$ bleached molecules, a ratio on the order of $1.76 \times 10^{6}$. For $80 \%$ bleaching and the approximately equivalent background of ND 3 , the ratio would be $4.8 \times 10^{4}$, and it would decrease further at even higher background intensities and bleaching levels to an asymptotic value on the order of $10^{3}$.

In these examples for 30 and $80 \%$ bleaching the thresholds are raised by almost 60 and 5000 times, respectively, and the saturated responses are compressed to about 0.4 and 0.2 , respectively, of the dark-adapted value.

Rhodopsin may be regarded as a catalyst for the transducinassociated exchange of GTP for GDP, which is an early step in transduction (Stryer, 1985). The rise in threshold with background therefore implies a greatly reduced forward effect of the catalyst with increasing concentration. This could be the case if, for example, as a consequence of photon absorption, the rhodopsin were to activate a reverse, as well as a forward, reaction. Since the response is known to be speeded up in the presence of a background, this is a possibility.

Bleaching does not speed up the response like backgrounds.
Also, the large differences in the number of photolyzed molecules in the bleached conditions compared to the equivalent background seem to imply different, as yet unknown, mechanisms of adaptive control.

\section{References}

Backstrom, A.-C., and S. O. Hemila (1979) Dark adaptation in frog rods: Changes in the stimulus-response function. J. Physiol. (Lond.) 287: $107-125$.

Barlow, H. B., and J. M. B. Sparrock (1964) The role of afterimages in dark adaptation. Science 144: 1309-1314.

Baylor, D. A., T. D. Lamb, and K.-W. Yau (1979) Responses of retinal rods to single photons. J. Physiol. (Lond.) 288: 613-634.

Baylor, D. A., G. Matthews, and K.-W. Yau (1980) Two components of electrical dark noise in toad retinal rod outer segments. J. Physiol. (Lond.) 309: 591-621.

Baylor, D. A., B. J. Nunn, and J. L. Schnapf (1984) The photocurrent, noise and spectral sensitivity of rods of the monkey Macca fascicularis. J. Physiol. (Lond.) 357: 575-607.

Blakemore, C. B., and W. A. H. Rushton (1965) Dark adaptation and increment threshold in a rod monochromat. J. Physiol. (Lond.) I8I. 612-628.

Boynton, R. M., and D. N. Whitten (1970) Visual adaptation in monkey cones: Recordings of late receptor potentials. Science 170:14231426.

Clack, J. W., and D. R. Pepperberg (1982) Desensitization of skate photoreceptors by bleaching and background light. J. Gen. Physiol. 80: 863-883.

Cornwall, M. C., A. Fein, and E. F. MacNichol (1983) Spatial localization of bleaching adaptation in isolated vertebrate rod photoreceptors. Proc. Natl. Acad. Sci. USA 80: 2785-2788.

Crawford, B. H. (1947) Visual adaptation in relation to brief conditioning stimuli. Proc. R. Soc. London [Biol.] 134: 283-302.

DeVries, H. (1943) The quantum character of light and its bearing upon the threshold of vision, the differential sensitivity, and acuity of the eye. Physica 10: 553-564.

Dowling, J. E. (1960) Chemistry of visual adaptation in the rat. Nature 188: 114-118.

Dowling, J. E., and H. Ripps (1972) Adaptation in skate photoreceptors. J. Gen. Physiol. 60: 698-719.

Fain, G. L. (1976) Sensitivity of toad rods: Dependence on wavelength and background illumination. J. Physiol. (Lond.) 261: 71-101.

Gold, G. H. (1979) Photoreceptor coupling in retina of the toad Bufo marinus. J. Neurophysiol. 42: 311-328.

Grabowski, S. R., and W. R. Pak (1975) Intracellular recordings of rod responses during dark adaptation. J. Physiol. (Lond.) 247: $363-$ 391.

Hecht, S., S. Schlaer, and M. H. Pirenne (1942) Energy, quanta and vision. J. Gen. Physiol. 25: 819-840.

Kleinschmidt, J., and J. E. Dowling (1975) Intracellular recordings from Gecko photoreceptors during light and dark adaptation. J. Gen. Physiol. 66: 617-648.

Leibovic, K. N. (1971) On the retinal basis of visual adaptation. Kybernetik 9(3): 96-111.

Leibovic, K. N. (1972) Nervous System Theory, Academic, New York.

Leibovic, K. N. (1975) Bleaching of visual pigment in steady illumination. Biol. Cybern. 19: 127-130.

Leibovic, K. N. (1981) The basis for response compression and shift of the operating curve during rod light adaptation. Presented at the 11 th Meeting of the Society for Neuroscience.

Lcibovic, K. N. (1983a) Blcaching of the isolated retina. Biol. Cybern. 19: 109-114.

Leibovic, K. N. (1983b) Transduction mechanisms in photoreceptor adaptation. Biophys. J. 41: 2(2) M.PM.C3.

Leibovic, K. N. (1986) Adaptation, brightness perception and their correlation with photoreceptor responses. In Cognitive Science Society 8th Annual Conference Proceedings, pp. 245-259, Lawrence Erlbaum, Hillsdale, NJ.

Normann, R. A., and F. S. Werblin (1974) Control of retinal sensitivity: Light and dark adaptation of retinal rods and cones. J. Gen. Physiol. 63: 37-61.

Penn, R. D., and W. A. Hagins (1972) Kinetics of the photocurrent of retinal rods. Biophys. J. 12: 1073-1094. 
Pepperberg, D. K. (1984) Rhodopsin and visual adaptation: Analysis of photoreceptor thresholds in the isolated skate retina. Vision Res. 24: 357-366.

Pepperberg, D. R., P. K. Brown, M. Lurie, and J. E. Dowling (1978) Visual pigment and photoreceptor sensitivity in the isolated skate retina. J. Gen. Physiol. 71: 369-396.

Rose, A. (1942) The relative sensitivities of television pick-up tubes, photographic film, and the human eye. Proc. Inst. Rad. Engr. 30: 293-300.
Rushton, W. A. H. (1961) Rhodopsin measurement and dark adaptation in a subject deficient in cone vision. J. Physiol. (Lond.) 156 193-205.

Rushton, W. A. H. (1962) The Ferrier Lecture 1962: Visual adaptation. Proc. R. Soc. London [Biol.] 162: 20-46.

Stryer, L. (1985) Molecular design of an amplification cascade in vision. Biopolymers 24: 29-47.

Weinstein, G. W., R. R. Hobson, and J. E. Dowling (1967) Light and dark adaptation in isolated rat retina. Nature 215: 134-138. 